\title{
The casualty profile from the Manchester bombing 1996: a proposal for the construction and dissemination of casualty profiles from major incidents
}

\author{
Simon D Carley, Kevin Mackway-Jones
}

\begin{abstract}
Objective-To produce a casualty profile for the Manchester bombing and to illustrate the potential uses of such templates in major incident planning.

Methods-A retrospective review of casualty notes from the Manchester bombing. Results-A complete anonymous casualty profile for the Manchester bombing

is given with AIS90 coded injuries. The majority $(129,62 \%)$ of casualties sustained minor injuries from flying glass. A significant number of casualties (36, 18\%) presented with emotional distress or medical problems. A wide age range of casualties was involved. Few patients (19, 9\%) required admission to hospital. There were no deaths and no casualties sustained major trauma.

Conclusions-Casualty profiles may be useful in the planning and testing of health service major incident plans. Such information should be easily accessible to all emergency planners.

(F Accid Emerg Med 1997;14:76-80)
\end{abstract}

Keywords: casualty profile; Manchester bombing; major incident plans.

On the 15 th of June 1996 a bomb detonated in the centre of Manchester. It was the largest bomb ever detonated on the British mainland by the IRA. Although the immediate area had been cleared there were still several thousand people present in the city centre at the time of detonation. The bomb caused damage over a wide area, with over 200 people being injured up to half a mile from the site of the explosion.

Major incidents, although relatively rare events, present special problems for the emergency services. Their infrequent nature makes planning and the motivation for planning difficult to achieve. Although it is necessary to adopt an all-hazards approach to emergency planning, a lot can be learnt from an examination of past incidents. An appreciation of the demographics of casualties and their injuries from major incidents may be of value for assessing equipment, staffing, and organisational procedures when writing and testing plans. At present, information on the pattern of injuries sustained in past major incidents is lacking. We have assembled an anonymous
Table 1 Mechanism of injury for the casualties

\begin{tabular}{lll}
\hline Mechanism of injury & Number & Percentage \\
\hline Flying glass & 129 & $62 \%$ \\
Blunt trauma & 41 & $20 \%$ \\
Medical/psychological & 36 & $17 \%$ \\
Did not wait & 2 & $1 \%$ \\
\hline
\end{tabular}

casualty profile from the Manchester bombing on 15 June 1996 for use by all emergency planners.

\section{Methods}

A retrospective review of all emergency department notes was conducted in the nearest five hospitals to the scene of the explosion. This included one paediatric and four adult hospitals. Information was obtained for casualties attending the emergency departments on the day of the incident. Casualties presenting to hospitals on subsequent days were not included in the analysis. Casualties were included in the profile if their presenting complaint had been caused by, or precipitated by, the bombing. Injuries were classified according to the abbreviated injury score, 1990 edition, ${ }^{1}$ by the authors. Casualties presenting to the accident and emergency (A\&E) departments on the day of the incident with conditions not directly caused by the bomb were excluded from the profile.

The best possible description of injury was obtained from the casualty cards, although this was often quiet brief.

\section{Results}

A comprehensive list of the casualties is presented in the appendix. The two most serious injuries for each casualty have been recorded, together with the corresponding AIS90 code. ${ }^{1}$ A breakdown of the mechanism of injury is given in table 1 , and table 2 shows the disposal of the casualties. The figure shows the age range of casualties on which data were available.

\section{Table 2 Disposal of casualties}

\begin{tabular}{lll}
\hline Disposal & Number & Percentage \\
\hline Home & 186 & $89 \%$ \\
Admitted for observation & 10 & $5 \%$ \\
Admitted for operation & 8 & $4 \%$ \\
Transferred for operation & 1 & $0.5 \%$ \\
Did not wait & 2 & $1 \%$ \\
\hline
\end{tabular}




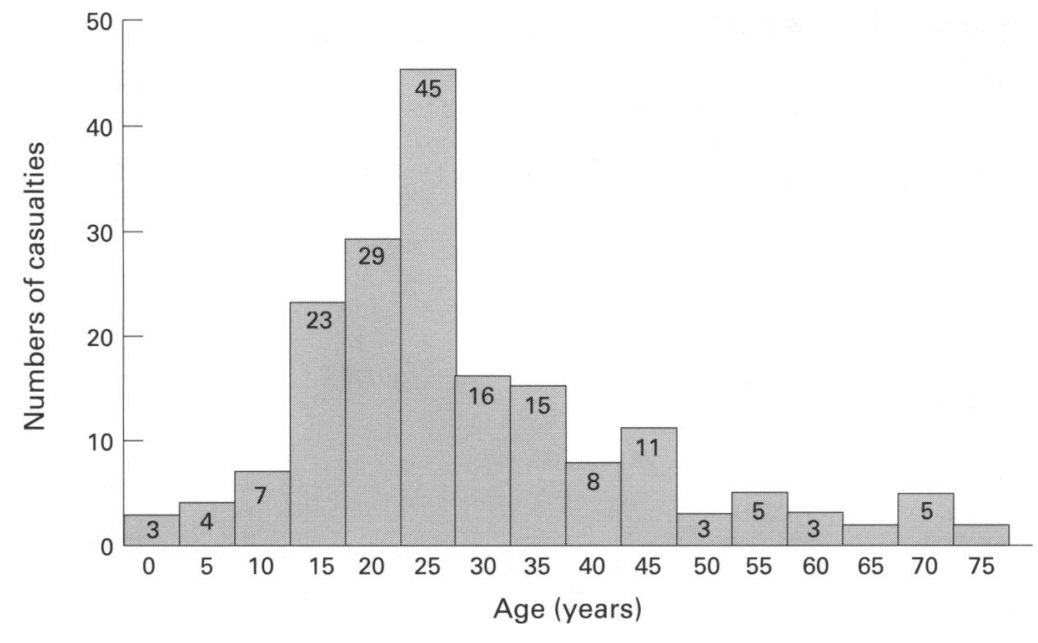

Age range of casualties. logy departments. Fifty per cent of casualties had some form of radiological investigation performed.

The vast majority $(89.4 \%)$ of casualties returned home from the $A \& E$ department, though 10 were admitted for observation and nine were admitted for subsequent operation (table 2). The receiving hospitals progressed little beyond the reception phase of their major incident plans, and there was no significant effect upon routine work in the receiving hospitals beyond the day of the incident itself.

Several papers have recorded brief descriptions of the severities of injury sustained in terrorist bombings, although many of these are now quite old. ${ }^{2-18}$ However, there is generally insufficient detail available on either the nature of the injuries sustained or the demographics of the casualties involved to aid planning.

It has previously been suggested that a database should be constructed for the description of each major incident to facilitate planning. ${ }^{15}$ We propose that the casualty profile for all major incidents be offered for publication in this journal soon after it occurs. Submission of a simple profile, as we have described, would take little effort by receiving hospitals. The exact structure of the profile may change with different types of incident (for example, burns) but the basic structure should remain the same.

Casualty profiles for different types of incidents will allow proper planning of staff equipment and support services during major incidents. Furthermore, hospital major incident plans should be tested on a regular basis, either as full incident scenarios with live casualty simulations or as table top exercises or PEWCs (Practical Exercise Without Casualties). In order to make such exercises as realistic as possible it is desirable that a realistic casualty profile is used.

\section{CONCLUSION}

Casualty profiles from real major incidents may be useful in planning and testing health service major incident plans. Detailed information on casualty profiles should be available to all professionals involved in emergency planning.

We would like to thank the consultants of the receiving hospitals for permission to review their patients' data.

\section{Appendix}

List of injuries and disposal

\begin{tabular}{|c|c|c|c|c|c|c|c|c|}
\hline & Age & $M O I$ & Injury 1 & AIS code 1 & Injury 2 & AIS code 2 & $X$ ray & Disposal \\
\hline 1 & & blunt & ?ear perforation & 240216.1 & & & $\mathbf{y}$ & home \\
\hline 2 & & blunt & abdo injury & 515099.9 & pregnant & & $\mathrm{n}$ & admitted \\
\hline 3 & & blunt & ankle strain & 850206.1 & & & $\mathbf{n}$ & home \\
\hline 4 & & blunt & back strain & 640678.1 & & & $\mathbf{y}$ & home \\
\hline 5 & 1 & blunt & head contusion & 110402.1 & & & $\mathbf{n}$ & home \\
\hline 6 & 11 & blunt & wrist fracture & 751800.2 & & & $\mathbf{y}$ & home \\
\hline 7 & 14 & blunt & knee contusion & 850802.1 & & & y & home \\
\hline 8 & 14 & blunt & leg abrasion & 810202.1 & & & $\mathrm{n}$ & home \\
\hline 9 & 14 & blunt & shoulder strain & 751020.1 & & & $\mathbf{n}$ & home \\
\hline 10 & 15 & blunt & arm contusion & 710402.1 & & & $\mathbf{n}$ & home \\
\hline 11 & 15 & blunt & facial contusions & 210402.1 & & & $\mathbf{n}$ & home \\
\hline 12 & 17 & blunt & knee strain & 850826.2 & emotional distress & & $\mathbf{n}$ & home \\
\hline 13 & 17 & blunt & neck strain & 640278.1 & & & $y$ & home \\
\hline
\end{tabular}




\begin{tabular}{|c|c|c|c|c|c|c|c|}
\hline & Age & $M O I$ & Injury 1 & AIS code 1 & Injury 2 & AIS code 2 & $X$ ray \\
\hline 14 & 18 & blunt & ? ear perforation & 240216.1 & & & $\mathbf{n}$ \\
\hline 15 & 19 & blunt & head contusion & 110402.1 & & & $\mathrm{y}$ \\
\hline 16 & 20 & blunt & head contusion & 110402.1 & & & $\mathrm{y}$ \\
\hline 17 & 22 & blunt & ankle strain & 850206.1 & & & $\mathrm{y}$ \\
\hline 18 & 22 & blunt & head laceration & 110602.1 & back strain & 640678.1 & $\mathrm{n}$ \\
\hline 19 & 23 & blunt & knee strain & 850826.2 & & & $\mathrm{y}$ \\
\hline 20 & 23 & blunt & neck strain & 640278.1 & back strain & 640678.1 & $\mathrm{y}$ \\
\hline 21 & 24 & blunt & back strain & 640478.1 & & & $\mathrm{n}$ \\
\hline 22 & 25 & blunt & head laceration & 110602.1 & & & $\mathrm{y}$ \\
\hline 23 & 26 & blunt & ? ear perforation & 240216.1 & & & $\mathrm{n}$ \\
\hline 24 & 27 & blunt & back contusion & 710602.1 & & & $\mathrm{y}$ \\
\hline 25 & 27 & blunt & back strain & 640678.1 & neck strain & 640278.1 & $\mathrm{n}$ \\
\hline 26 & 27 & blunt & head contusion & 110402.1 & & & $\mathrm{y}$ \\
\hline 27 & 29 & blunt & shoulder strain & 751020.1 & & & $\mathrm{y}$ \\
\hline 28 & 30 & blunt & ? ear perforation & 240216.1 & & & $\mathrm{n}$ \\
\hline 29 & 31 & blunt & neck strain & 640278.1 & & & $\mathrm{n}$ \\
\hline 30 & 32 & blunt & shoulder strain & 751020.1 & & & $\mathrm{y}$ \\
\hline 31 & 34 & blunt & ? ear perforation & 240216.1 & & & $\mathrm{n}$ \\
\hline 32 & 34 & blunt & abdominal contusion & 510402.1 & & & $\mathrm{y}$ \\
\hline 33 & 34 & blunt & back strain & 640678.1 & ankle strain & 850206.1 & $\mathrm{n}$ \\
\hline 34 & 35 & blunt & elbow sprain & 750620.1 & & & $\mathrm{y}$ \\
\hline 35 & 35 & blunt & facial laceration & 210602.1 & & & $\mathrm{n}$ \\
\hline 36 & 41 & blunt & back strain & 640678.1 & neck strain & 640278.1 & $\mathrm{y}$ \\
\hline 37 & 42 & blunt & head contusion & 110402.1 & & & $\mathrm{y}$ \\
\hline 38 & 45 & blunt & head laceration & 110602.1 & neck strain & 640278.1 & $\mathrm{y}$ \\
\hline 39 & 46 & blunt & neck strain & 640278.1 & & & $\mathrm{n}$ \\
\hline 40 & 56 & blunt & facial fracture & 250400.1 & & & $\mathrm{y}$ \\
\hline 41 & 57 & blunt & ? ear perforation & 240216.1 & & & $\mathrm{n}$ \\
\hline 42 & & dnw & unknown & & & & $\mathrm{n}$ \\
\hline 43 & 42 & dnw & unknown & & & & $\mathrm{n}$ \\
\hline 44 & & $\mathrm{fg}$ & back laceration & 410602.1 & arm laceration & 710602.1 & $\mathrm{y}$ \\
\hline 45 & & fg & facial laceration & 210602.1 & & & $\mathrm{y}$ \\
\hline 46 & & $\mathrm{fg}$ & facial laceration & 210602.1 & facial laceration & 210602.1 & $\mathrm{y}$ \\
\hline 47 & & fg & hand laceration & 710602.1 & & & $\mathrm{y}$ \\
\hline 48 & & fg & head laceration & 110602.1 & finger laceration & 710602.1 & $\mathrm{y}$ \\
\hline 49 & & $\mathrm{fg}$ & head laceration & 110602.1 & & & $\mathrm{n}$ \\
\hline 50 & & fg & leg laceration & 810602.1 & leg laceration & 810602.1 & $\mathrm{n}$ \\
\hline 51 & & fg & multiple lacerations & 910600.1 & & & $\mathrm{n}$ \\
\hline 52 & & fg & $\begin{array}{l}\text { tendon laceration, } \\
\text { hand }\end{array}$ & 740200.1 & & & $\mathrm{y}$ \\
\hline 53 & 1 & fg & finger laceration & 710602.1 & wrist laceration & 710602.1 & $\mathbf{y}$ \\
\hline 54 & 1 & fg & head laceration & 110602.1 & & & $\mathrm{n}$ \\
\hline 55 & 4 & $\mathrm{fg}$ & eye & 240602.1 & & & $\mathrm{n}$ \\
\hline 56 & 6 & fg & arm laceration & 710602.1 & knee laceration & 810602.1 & $\mathrm{y}$ \\
\hline 57 & 6 & fg & head laceration & 110602.1 & & & $\mathrm{y}$ \\
\hline 58 & 7 & $\mathrm{fg}$ & leg laceration & 810602.1 & thigh laceration & 810602.1 & $\mathrm{n}$ \\
\hline 59 & 11 & fg & head laceration & 110602.1 & & & $\mathrm{n}$ \\
\hline 60 & 12 & $\mathrm{fg}$ & eye & 240602.1 & & & $\mathrm{n}$ \\
\hline 61 & 12 & fg & eye & 240602.1 & & & $\mathbf{y}$ \\
\hline 62 & 12 & $\mathrm{fg}$ & facial laceration & 210602.1 & & & $\mathbf{y}$ \\
\hline 63 & 12 & $\mathrm{fg}$ & finger laceration & 710602.1 & & & $\mathrm{y}$ \\
\hline 64 & 12 & $\mathrm{fg}$ & leg laceration & 810602.1 & & & $\mathrm{y}$ \\
\hline 65 & 13 & fg & thigh laceration & 810602.1 & calf laceration & 810602.1 & $\mathrm{y}$ \\
\hline 66 & 14 & fg & arm laceration & 710602.1 & & & $\mathrm{y}$ \\
\hline 67 & 14 & $\mathrm{fg}$ & eye & 240602.1 & & & $\mathrm{n}$ \\
\hline 68 & 14 & fg & hand laceration & 710602.1 & thumb laceration & 710602.1 & $\mathrm{y}$ \\
\hline 69 & 14 & fg & head laceration & 110602.1 & hand laceration & 710602.1 & $\mathrm{y}$ \\
\hline 70 & 14 & fg & knee laceration & 810602.1 & hand laceration & 710602.1 & $\mathrm{y}$ \\
\hline 71 & 14 & fg & thumb laceration & 710602.1 & & & $\mathrm{y}$ \\
\hline 72 & 15 & $\mathrm{fg}$ & eye & 240602.1 & & & $\mathrm{n}$ \\
\hline 73 & 15 & fg & foot laceration & 810602.1 & & & $\mathrm{n}$ \\
\hline 74 & 16 & fg & back laceration & 410602.1 & finger laceration & 710602.1 & $\mathrm{y}$ \\
\hline 75 & 16 & fg & leg laceration & 810602.1 & arm laceration & 710602.1 & $\mathrm{y}$ \\
\hline 76 & 17 & fg & calf laceration & 810602.1 & & & $\mathrm{y}$ \\
\hline 77 & 17 & $\mathrm{fg}$ & head laceration & 110602.1 & & & $\mathrm{y}$ \\
\hline 78 & 18 & $\mathrm{fg}$ & arm laceration & 710802.1 & & & $\mathrm{y}$ \\
\hline 79 & 18 & fg & finger laceration & 710602.1 & & & $\mathrm{n}$ \\
\hline 80 & 19 & $\mathrm{fg}$ & hand laceration & 710602.1 & & & $\mathrm{y}$ \\
\hline 81 & 19 & fg & head laceration & 110602.1 & & & $\mathrm{y}$ \\
\hline 82 & 19 & fg & head laceration & 110602.1 & & & $\mathrm{y}$ \\
\hline 83 & 20 & fg & arm laceration & 710602.1 & & & $\mathrm{y}$ \\
\hline 84 & 20 & fg & back laceration & 410602.1 & & & $\mathrm{n}$ \\
\hline 85 & 20 & fg & eye & 240602.1 & & & $\mathrm{n}$ \\
\hline 86 & 20 & fg & head laceration & 110602.1 & & & $\mathrm{n}$ \\
\hline 87 & 20 & fg & thumb laceration & 710602.1 & finger laceration & 710602.1 & $\mathrm{y}$ \\
\hline 88 & 20 & fg & unknown & & & & $\mathrm{n}$ \\
\hline 89 & 21 & fg & back laceration & 410602.1 & & & $\mathrm{n}$ \\
\hline 90 & 21 & fg & head laceration & 110602.1 & & & $\mathrm{y}$ \\
\hline 91 & 22 & fg & hand laceration & 710602.1 & & & $\mathrm{y}$ \\
\hline 92 & 22 & $\mathrm{fg}$ & head laceration & 110604.2 & & & $\mathrm{y}$ \\
\hline 93 & 22 & fg & leg laceration & 810602.1 & & & $\mathrm{n}$ \\
\hline 94 & 22 & fg & leg laceration & 810602.1 & hand laceration & 710602.1 & $\mathrm{y}$ \\
\hline 95 & 22 & $\mathrm{fg}$ & leg laceration & 810602.1 & & & $\mathrm{y}$ \\
\hline 96 & 22 & fg & thumb laceration & 710602.1 & & & $\mathrm{y}$ \\
\hline 97 & 23 & $\mathrm{fg}$ & ankle laceration & 810602.1 & & & $\mathrm{y}$ \\
\hline 98 & 23 & $\mathrm{fg}$ & back laceration & 410602.1 & & & $\mathrm{n}$ \\
\hline 99 & 23 & fg & facial laceration & 210602.1 & & & $\mathbf{y}$ \\
\hline 100 & 23 & fg & hand laceration & 710602.1 & & & $\mathrm{y}$ \\
\hline 101 & 23 & fg & leg laceration & 810600.1 & & & $\mathrm{y}$ \\
\hline 102 & 23 & fg & shoulder laceration & 710600.1 & & & $\mathrm{y}$ \\
\hline 103 & 24 & $\mathrm{fg}$ & arm laceration & 710602.1 & thigh laceration & 810602.1 & $\mathrm{n}$ \\
\hline
\end{tabular}




\begin{tabular}{|c|c|c|c|c|c|c|}
\hline & Age & $M O I$ & Injury 1 & AIS code 1 & Injury 2 & AIS code 2 \\
\hline 104 & 24 & $\mathrm{fg}$ & head laceration & 110602.1 & facial laceration & 210602.1 \\
\hline 105 & 24 & fg & multiple lacerations & 910600.1 & & \\
\hline 106 & 24 & fg & shoulder laceration & 710602.1 & & \\
\hline 107 & 25 & fg & arm laceration & 710602.1 & & \\
\hline 108 & 25 & fg & facial laceration & 210602.1 & ear avulsion & 210800.1 \\
\hline 109 & 25 & fg & facial laceration & 210604.2 & & \\
\hline 110 & 25 & fg & head laceration & 110602.1 & back laceration & 410602.1 \\
\hline 111 & 25 & fg & head laceration & 110602.1 & & \\
\hline 112 & 25 & fg & leg laceration & 810600.1 & & \\
\hline 113 & 25 & fg & leg laceration & 810602.1 & shoulder laceration & 710602.1 \\
\hline 114 & 25 & fg & neck laceration & 310602.1 & thigh laceration & 810602.1 \\
\hline 115 & 26 & fg & arm laceration & 710600.1 & & \\
\hline 116 & 26 & fg & arm laceration & 710602.1 & hand laceration & 710602.1 \\
\hline 117 & 26 & fg & back laceration & 410602.1 & hand laceration & 710602.1 \\
\hline 118 & 26 & fg & finger laceration & 710602.1 & elbow laceration & 710602.1 \\
\hline 119 & 26 & fg & head laceration & 110602.1 & arm laceration & 710602.1 \\
\hline 120 & 26 & fg & head laceration & 110602.1 & head contusion & 110402.1 \\
\hline 121 & 26 & fg & leg laceration & 810602.1 & & \\
\hline 122 & 26 & fg & thigh laceration & 810602.1 & arm laceration & 710602.1 \\
\hline 123 & 27 & fg & arm laceration & 710602.1 & leg laceration & 810602.1 \\
\hline 124 & 27 & fg & finger laceration & 710602.1 & head laceration & 110602.1 \\
\hline 125 & 27 & fg & finger laceration & 710602.1 & ankle laceration & 810602.1 \\
\hline 126 & 27 & fg & head laceration & 110602.1 & & \\
\hline 127 & 27 & fg & head laceration & 110602.1 & finger laceration & 710602.1 \\
\hline 128 & 28 & fg & head laceration & 110602.1 & & \\
\hline 129 & 29 & fg & head laceration & 110602.1 & back laceration & 410602.1 \\
\hline 130 & 29 & fg & leg laceration & 810602.1 & & \\
\hline 131 & 29 & fg & tendon laceration, leg & 840200.2 & & \\
\hline 132 & 30 & fg & back laceration & 410602.1 & finger fracture & 752404.1 \\
\hline 133 & 31 & fg & head laceration & 110602.1 & arm laceration & 710602.1 \\
\hline 134 & 32 & fg & foot laceration & 810602.1 & & \\
\hline 135 & 32 & fg & head laceration & 110600.1 & hand laceration & 710600.1 \\
\hline 136 & 32 & fg & leg laceration & 810602.1 & & \\
\hline 137 & 32 & fg & wrist laceration & 710602.1 & knee laceration & 810602.1 \\
\hline 138 & 33 & fg & ankle laceration & 810602.1 & & \\
\hline 139 & 34 & fg & ankle laceration & 810602.1 & & \\
\hline 140 & 34 & fg & hand laceration & 710602.1 & & \\
\hline 141 & 35 & fg & ankle laceration & 810602.1 & & \\
\hline 142 & 35 & fg & arm laceration & 710602.1 & thumb laceration & 710602.1 \\
\hline 143 & 36 & fg & back laceration & 410602.1 & & \\
\hline 144 & 36 & fg & facial laceration & 210602.1 & thigh laceration & 810602.1 \\
\hline 145 & 36 & fg & thigh laceration & 810602.1 & calf laceration & 810602.1 \\
\hline 146 & 37 & fg & calf laceration & 810602.1 & thigh laceration & 810602.1 \\
\hline 147 & 40 & fg & back laceration & 410602.1 & & \\
\hline 148 & 42 & fg & face degloving & 210804.2 & hand tendon & 740200.1 \\
\hline 149 & 42 & fg & leg laceration & 810600.1 & & \\
\hline 150 & 42 & fg & leg laceration & 810600.1 & & \\
\hline 151 & 42 & fg & leg laceration & 810600.1 & & \\
\hline 152 & 43 & fg & facial laceration & 210602.1 & & \\
\hline 153 & 43 & fg & head laceration & 110602.1 & facial laceration & 210602.1 \\
\hline 154 & 44 & fg & leg laceration & 810602.1 & head laceration & 110602.1 \\
\hline 155 & 45 & fg & facial laceration & 210602.1 & & \\
\hline 156 & 46 & fg & back laceration & 410602.1 & & \\
\hline 157 & 46 & fg & elbow laceration & 710602.1 & & \\
\hline 158 & 46 & fg & head laceration & 110602.1 & & \\
\hline 159 & 48 & fg & head laceration & 110602.1 & back laceration & 410602.1 \\
\hline 160 & 49 & fg & digital nerve & 730299.1 & finger laceraation & 710602.1 \\
\hline 161 & 54 & fg & leg laceration & 810602.1 & arm laceration & 710602.1 \\
\hline 162 & 56 & fg & head laceration & 110602.1 & & 710602.1 \\
\hline 163 & 59 & fg & head laceration & 110602.1 & abdo laceration & 510602.1 \\
\hline 164 & 60 & fg & head laceration & 110602.1 & & \\
\hline 165 & 63 & fg & hand laceration & 710602.1 & & \\
\hline 166 & 64 & fg & head laceration & 110602.1 & ankle laceration & 810602.1 \\
\hline 167 & 68 & fg & hand laceration & 710602.1 & & \\
\hline 168 & 69 & $\mathrm{fg}$ & facial laceration & 210602.1 & facial laceration & 210602.1 \\
\hline 169 & 70 & fg & arm laceration & 710602.1 & head contusion & 110402.1 \\
\hline 170 & 70 & fg & hand laceration & 710602.1 & head laceration & 110602.1 \\
\hline 171 & 71 & fg & arm laceration & 710602.1 & wrist laceration & 710602.1 \\
\hline 172 & 74 & fg & leg laceration & 810602.1 & & \\
\hline 173 & & na & angina & & & \\
\hline 174 & & na & asthma & & & \\
\hline 175 & & na & diabetic & & & \\
\hline 176 & & na & emotional distress & & & \\
\hline 177 & & na & emotional distress & & & \\
\hline 178 & & na & emotional distress & & & \\
\hline 179 & & na & emotional distress & & & \\
\hline 180 & & na & emotional distress & & & \\
\hline 181 & & na & emotional distress & & & \\
\hline 182 & & na & emotional distress & & & \\
\hline 183 & & na & emotional distress & & & \\
\hline 184 & & na & emotional distress & & & \\
\hline 185 & & na & headache & & & \\
\hline 186 & 14 & na & emotional distress & & & \\
\hline 187 & 17 & na & ? labour & & & \\
\hline 188 & 17 & na & emotional distress & & & \\
\hline 189 & 18 & na & emotional distress & & & \\
\hline 190 & 18 & na & emotional distress & & & \\
\hline 191 & 18 & na & emotional distress & & & \\
\hline 192 & 20 & na & emotional distress & & & \\
\hline 193 & 21 & na & faint & & & \\
\hline 194 & 23 & na & diabetic & & & \\
\hline
\end{tabular}




\begin{tabular}{|c|c|c|c|c|c|c|c|c|}
\hline & Age & $M O I$ & Injury 1 & AIS code 1 & Injury 2 & AIS code 2 & $X$ ray & Disposal \\
\hline 195 & 23 & na & emotional distress & & & & $\mathbf{n}$ & home \\
\hline 196 & 24 & na & emotional distress & & & & $\mathbf{n}$ & home \\
\hline 197 & 25 & na & asthmatic & & & & $\mathbf{n}$ & home \\
\hline 198 & 26 & na & diabetic & & & & $\mathbf{n}$ & home \\
\hline 199 & 26 & na & emotional distress & & & & $\mathbf{n}$ & home \\
\hline 200 & 28 & na & emotional distress & & & & $\mathbf{n}$ & home \\
\hline 201 & 29 & na & emotional distress & & & & $\mathbf{n}$ & home \\
\hline 202 & 34 & na & epilepsy & & & & $\mathrm{n}$ & home \\
\hline 203 & 43 & na & angina & & & & $\mathbf{n}$ & admitted \\
\hline 204 & 47 & na & emotional distress & & & & $\mathbf{n}$ & home \\
\hline 205 & 52 & na & emotional distress & & & & $\mathbf{n}$ & home \\
\hline 206 & 56 & na & angina & & & & $\mathrm{y}$ & admitted \\
\hline 207 & 58 & na & asthma & & & & $\mathrm{n}$ & home \\
\hline 208 & 74 & na & emotional distress & & & & $\mathbf{n}$ & home \\
\hline
\end{tabular}

y,yes; n,no; MOI,mechanism of injury; fg,flying glass; blunt,blunt trauma; dnw,did not wait; na,not applicable.

1 American Association for Automotive Medicine. The Abbreviated Injury Scale (AIS) 1990 revision. Des Plaines, Illinois: AAAM, 1990.

2 Cooper GJ. Casualties from terrorist bombings. J Trauma 1983;23:955-67.

3 Adler J. Terrorist bombing experience during 1975-79. Isr J Med Sci 1983;19:189-93.

4 Brismar B, Bergenwald L. The terrorist bomb explosion in Bologna, Italy, 1980: an analysis of the effects and the injuries sustained. J Trauma 1982;22:216-20.

5 Hadden W, Rutherford W, Merret XX. The injuries of terrorist bombing : a study of 1532 consecutive patients. $\mathrm{Br}$ J Surg 1978;65:525-31.

6 Tucker K, Lettin A. The tower of London bomb explosion BMJ 1975;iii:287-89.

7 Kennedy TL, Johnston GW. Civilian bomb injuries. BMJ 1975;i:382-3.

8 Scott BA, Fletcher JR, Pulliam MW, et al. The Beirut terrorist bombing. Br J Neurosurg 1986;1:107-10.

Caro $\mathrm{D}$, Irving $\mathrm{M}$. The Old Bailey bomb explosion. Lancet 1973;ii:1433-5.

10 Waterworth TA, Carr MJT. Report on injuries sustained by patients treated at the Birmingham General Hospital patients treated at the Birmingham General Hospital Rignault DP, Deligny MC. The 1986 terrorist bombing experience in Paris. Ann Surg 1988;209:368-73.

12 Frykberg ER, Tepas J. Terrorist bombings. Ann Surg 1988; 208:569-76.

13 Rutherford W. Civil disturbances in Belfast. Injury 1972;4: 189-99.

14 Brown MG, Marshall SG. The Enniskillin bomb: a disaster plan. BMJ 1988;297:1113-6.

15 Rutherford WH. Experience in the Accident and Emergency department of the Royal Victoria Hospital with patients from civil disturbances in Belfast 1969-1972, with a review of disasters in the United Kingdom 1951-1971. Injury 1972;4:189-99.

16 Hodgetts TJ. Lessons from the Musgrave Park Hospital bombing. Injury 1993;24:219-21.

17 Johnstone DJ, Evans SC, Field RE, Booth SJ. The Victoria bomb: a report from the Westminster Hospital. Injury 1993;24:5-9.

18 Mallone S, Shariat S, Stennies G, Waxweiler R, Hogan D, Jordan F. Physical injuries and fatalities resulting from the Oklahoma City bombing. JAMA 1996;276:382-7.

\section{British Association of Plastic Surgeons Advanced course in plastic surgery 6:2}

The sixth meeting will be held on 4-5 April 1997 in Sheffield:

\section{Hand 1. Trauma/peripheral nerve surgery}

The course is aimed at consultants and trainees in plastic surgery but members of the British Association for Accident and Emergency Medicine are invited to attend. The fee will be $£ 350.00$, which includes one night's accommodation and all meals.

Further details and an application form are available from the British Association of Plastic Surgeons, The Royal College of Surgeons, 35-43 Lincoln's Inn Fields, London WC2A 3PN. Tel 0171831 5161/2; fax 0171 8314041. 